\title{
Option Pricing with Markov Switching
}

\author{
Cheng-Der Fuh ${ }^{1}$, Kwok Wah Remus $\mathrm{Ho}^{2}$, Inchi $\mathrm{Hu}^{3}$ and Ren-Her Wang ${ }^{4 *}$ \\ ${ }^{1}$ National Central University, ${ }^{2}$ Chinese University of Hong Kong, \\ ${ }^{3}$ Hong Kong University of Science and Technology and ${ }^{4}$ Tamkang University
}

\begin{abstract}
In this article, we consider a model of time-varying volatility which generalizes the classical Black-Scholes model to include regime-switching properties. Specifically, the unobservable state variables for stock fluctuations are modeled by a Markov process, and the drift and volatility parameters take different values depending on the state of this hidden Markov process. We provide a closed-form formula for the arbitrage-free price of the European call option, when the hidden Markov process has finite number of states. Two simulation methods, the discrete diffusion method and the Markovian tree method, for computing the European call option price are presented for comparison.
\end{abstract}

Key words: Arbitrage, hidden Markov model, implied volatility, Laplace transform, Markovian tree.

\section{Introduction}

It is well-known that the volatility of financial time series changes over time and the changes tend to be persistent. Furthermore, some stylized features of the volatility have been established by many empirical studies. These features include volatility clustering, leverage effects, higher volatility following non-trading periods, higher volatility after foreseeable releases of important information, and co-movements in volatility, etc. Therefore, models of changing volatility have been developed to capture these empirical phenomena. Three classes of models that received considerable attention are $\mathrm{ARCH}$ type models, stochastic volatility models, and regime switching models.

The ARCH type models was first proposed by (Engle, 1982) and later generalized to GARCH by (Bollerslev, 1986; 1987). Option pricing in GARCH models was first investigated by (Duan, 1995). Option pricing in stochastic volatility model were considered by (Hull and White, 1987), (Stein and Stein, 1991), (Wiggins, 1987) and (Heston, 1993). Uncertain volatility was studied in (Avellaneda,

\footnotetext{
${ }^{*}$ Corresponding author.
} 
Levy and Parás, 1995). The Markov switching model of (Hamilton, 1988; 1989) is capable of accommodating time-varying volatility. (Hamilton and Susmel, 1994) proposed the Markov switching ARCH model. In a partial equilibrium model, (Turner, Startz and Nelson, 1989) formulated a switching model for excess returns, in which returns switch exogenously between a Gaussian low variance regime and a Gaussian high variance regime. (So, Lam and Li, 1998) generalized the stochastic volatility model to incorporate Markov regime switching properties. (David, 1997) studied the regime switching properties of the drift in the classical Cox-Ingersoll-Ross model. (Veronesi, 1999) consider Gaussian diffusion model with drift depending on the hidden states. (Di Masi, Kabanov and Runggladier, 1994) considered the problem of hedging a European call option for a diffusion model, where drift and volatility are functions of a two-state Markov process. (Guo, 2001) considered the same model and gave a closed-form formula for the European call option, which contains a couple of mistakes. (Duan, Popova and Ritchken, 2002) developed a family of option pricing models when the underlying stock price dynamic is modeled by a regime switching process. The family included Markov switching model of (Hamilton, 1989) and extended GARCH option models as a special limiting case.

Recent research (cf. Bittlingmayer, 1998) has shown that investors' uncertainty over some important factors affecting the economy may greatly impact the volatility of stock returns. More generally, there is evidence that investors tend to be more uncertain about the future growth rate of the economy during recessions, and thereby partially justifying a higher volatility of stock returns. (Veronesi, 1999) demonstrated that stock prices overreact to bad news in good time and underreact to good news in bad times. The empirical evidence of (Maghrebi, Kim and Nishina, 2007) suggested that Markov regime switching models are regime dependencies to adjust forecast errors. The nonlinearities in volatility expectations can be captured by Markov regime switching models.

In this paper, to encompass the empirical phenomena of stock fluctuations related to the business cycle, we introduce a model of an incomplete market by adjoining the Black-Scholes exponential Brownian motion model with a hidden Markov process. Specifically, we assume that stock prices are generated by realization of a Gaussian diffusion process, and that the drift and volatility parameters take different values depending on the state of a hidden Markov process. That is, we assume that investors cannot observe the drift rates, nor the volatility of the process, and they have to infer them from their observations. We call this model a Black-Scholes model with Markov switching, or a hidden Markov model (HMM) in brief. The contribution of this paper is to provide a close-form formula for the arbitrage-free price of the European call option when the hidden Markov process has finite number of states. Two simulation methods, the discrete diffu- 
sion method and the Markovian tree method, for computing the European call option price are presented for comparison.

This article is organized as follows. In Section 2, we describe the BlackScholes model with Markov switching that capture the phenomenon of business cycles in stock fluctuations. Then, we provide a closed-form formula for the price of the European call option in Section 3. In particular, we give an explicit analytic formula for the option price in a finite-state HMM. To illustrate the performance of the formula, in Section 4, we present results obtained from the discrete diffusion method and the Markovian tree method for comparison. Section 5 gives conclusions. The derivation of the option price formula is given in the Appendix.

\section{The Hidden Markov Model and Option Price}

Consider the following model for the fluctuations of a single stock price $X_{t}$, which incorporates the business cycle,

$$
d X_{t}=X_{t} \mu_{\varepsilon(t)} d t+X_{t} \sigma_{\varepsilon(t)} d W_{t}
$$

where $\varepsilon(t)$ is a stochastic process representing the state of the business cycle, and $W_{t}$ is the standard Wiener process, which is independent of $\varepsilon(t)$. For each state of $\varepsilon(t)$, the drift parameter $\mu_{\varepsilon(t)}$ and volatility parameter $\sigma_{\varepsilon(t)}$ take different values when $\varepsilon(t)$ is in different states.

We also assume that the total shares of the risky asset is fixed and normalized to 1 . The risk-free asset has an instantaneous rate of return equal to $r$. Note that this assumption not only simplifies the analysis for option pricing, but also matches the empirical finding that the volatility of the risk-free rate is much lower than the volatility of market returns.

Assume that $\varepsilon(t)$ is a Markov process with a finite number of states. In practice, a three-state HMM is rich enough to capture the empirical phenomena of most financial time series. Thus we will focus our discussion on the case where the number of states equals three, although our method can be applied to arbitrary number of states. A business cycle can be divided into three different states, expansion, transition, and contraction. A growing economy is described as being in expansion. In this state, let $\varepsilon(t)=2, \mu_{\varepsilon(t)}=\mu_{2}$ and $\sigma_{\varepsilon(t)}=\sigma_{2}$. Similarly we use $\varepsilon(t)=1,0$ to denote transition state, and contraction state, respectively, with corresponding drifts $u_{\varepsilon(t)}=\mu_{1}, \mu_{0}$ and volatilities $\sigma_{\varepsilon(t)}=\sigma_{1}, \sigma_{0}$. More generally, we can use the state space $\Omega=\{0,1, \cdots, N\}$ for $\varepsilon(t)$ to model more complex business cycle structures. In this section, for simplicity, we consider a three-state HMM for a single stock price $X_{t}$ by using $(2.1)$, where $\varepsilon(t)$ is a Markov 
process representing the state of the business cycle. Let

$$
\varepsilon(t)= \begin{cases}0, & \text { when the business cycle is in contraction, } \\ 1, & \text { when the business cycle is in transition, } \\ 2, & \text { when the business cycle is in expansion }\end{cases}
$$

The three-state model will extend to finite $n$-state $(n \geq 3)$ model similarly. The two-state model can not be extended easily to $n$-state model. Because the twostate model has a particular property that switches to one another state. The $n$-state model may switch multiple states. The method of two-state model can not completely imitate to three-state or $n$-state model.

In the preceding model, we assume that each state has different volatility. It is conceivable that, sometimes, investors conduct their buying and selling in such a way that the change of volatilities is not detectable; that is, $\sigma$ are identical (e.g., (David, 1997) and (Veronesi, 1999)). When $\sigma$ remains unchanged, it is difficult to detect the state change of $\varepsilon(t)$. It is plausible that a change of state in a business cycle, will manifest itself in both stochastic volatility and drift. If we assume that the volatility in different states are distinct, then without loss of generality we can assume that $\varepsilon(t)$ is actually observable since the local quadratic variation of $X_{t}$ in any small interval to the left of $t$ will yield $\sigma_{\varepsilon(t)}$ exactly; see e.g., ((McKean, 1969); (Hamilton, 1988; 1989); (Hamilton and Susmel, 1994); (Guo, 2001); (Duan et al., 2002)). That is, the filtration $\mathcal{F}^{X}$ generated by the process $\left\{X_{t}\right\}$ contains the filtration $\mathcal{F}^{\varepsilon}$ generated by $\{\varepsilon(t)\}$.

With regard to the transition rate among different states, let $\lambda_{i}$ be the rate of leaving state $i$, and let $\tau_{i}$ be the time of leaving state $i$. We assume that

$$
P\left(\tau_{i}>t\right)=e^{-\lambda_{i} t}, \quad i=0,1,2 .
$$

The exponential holding time is a crucial assumption that leads to the closed form formula of option prices. The closed form formula not only facilitates the computation of option prices but also allows a valuable understanding of how different components of the model affect option prices. It is well known that the exponential holding time gives rise to the memoryless property. In additional to the mathematical tractability of the exponential holding time, the memoryless property is reasonable from a practical standpoint. Unless we have a definite theory on the distribution of $\tau_{i}$, we may assume uniform ignorance, that is, no matter how long in the current state, we are equally uncertain about the time for the next change of state.

Despite the success of the classical Black-Scholes model, some empirical phenomena have received much attention recently. Important assumptions in the Black-Scholes model are that the underlying asset distribution is lognormal and that the volatility is a fixed constant. However, empirical evidence suggests that 
the asset distribution exhibits leptokurtic and unsymmetrical features and the volatility has a clustering phenomenon. By adjoining the Black-Scholes exponential Brownian motion with a hidden Markov process (the drift and volatility parameters take different values depending on the state of this hidden Markov process), model (2.1) displays the asymmetric leptokurtic features, negative skewness, and negative correlation with future volatility (cf. David, 1997). (Veronesi, 1999) showed that Markov switching model is better than the Black-Scholes model in explaining the features of stock returns, including volatility clustering, leverage effects, excess volatility and time-varying expected returns.

Model (2.1) is incomplete (cf. (Harrison and Pliska, 1981); (Harrison and Kreps, 1979)) because the stock price is not only driven by the Wiener process $W$ alone but also by the hidden Markov process $\varepsilon(t)$. One way to deal with this situation was given by (Föllmer and Sondermann, 1986), and (Schweizer, 1991), who used the idea of hedging under a mean-variance criterion. Here, we use the following approach to complete the market. At each time $t$, there is a market for a security that pays one unit of account (say, a dollar) at the next time $\tau(t)=\inf \{u>t \mid \varepsilon(u) \neq \varepsilon(t)\}$ when the Markov chain $\varepsilon(t)$ changes state. One can think of this as an insurance contract that compensates its holder for any losses due to the next state change. If one wants to hedge a given deterministic loss $C$, one can hold $C$ units of the current change-of-state (COS) contracts.

The absence of arbitrage is effectively the same as the existence of a riskneutral probability $Q$, equivalent to $P$, under which the price of any derivative is the expected discounted value of its future cash flow. With COS contracts, the transition rate of the Markov chain under the risk neutral measure $Q$, which is different from the real transition rate, can be identified. We now proceed to derive the relationship between the real transition rate $\lambda$ and the risk-neutral transition rate $\lambda^{Q}$ under measure $Q$. Denote by $\tau(t)=\inf \{u>t \mid \varepsilon(u) \neq \varepsilon(t)\}$ the first change time after $t$ the Markov chain $\varepsilon(u)$ changes state. Using the exponential holding time assumption, we have

$$
E\left[e^{-\left(r+k_{\varepsilon(t)}\right)(\tau(t)-t)} \mid \varepsilon(t)=i\right]=\frac{\lambda_{i}}{r+k_{i}+\lambda_{i}}=\frac{\lambda_{i}^{Q}}{r+\lambda_{i}^{Q}}=E^{Q}\left[e^{-r(\tau(t)-t)} \mid \varepsilon(t)=i\right],
$$

where $k_{\varepsilon(t)}$ can be thought of as a risk-premium coefficient. Thus

$$
\lambda_{i}^{Q}=\frac{r \lambda_{i}}{r+k_{i}}
$$

Hereafter, we will only use the transition rate $\lambda^{Q}$ under $Q$. When there is no danger of confusion, we will drop the $Q$ and use $\lambda$ as the transition rate under $Q$ for simplicity. 
Applying the same analysis to the underlying risky-asset implies that its price process $X$ must have the form

$$
\frac{d X_{t}}{X_{t}}=r d t+\sigma_{\varepsilon(t)} d W_{t}^{Q}
$$

where $W_{t}^{Q}$ is the standard Brownian motion under the risk-neutral probability $Q$.

We first present the following theorem for a two-state HMM because of its simplicity. Let $T_{i}$ be occupation time of state 0 , when the chain starts from state $i$. That is the total amount of time between 0 and $T$ during which $\varepsilon(t)=0$, starting from state $i$ for $i=0,1$. Let $f_{i}(t, T)$ be the probability distribution function of $T_{i}$. The proof of Theorem 1 is given in the Appendix.

Theorem 1. Under HMM (2.1) and (2.2), COS, and the riskless interest rate $r$, the arbitrage free price of a European call option with expiration date $T$ and strike price $K$ is given by

$$
\begin{aligned}
V_{i}(T, K, r) & =E^{Q}\left[e^{-r T}\left(X_{T}-K\right)^{+} \mid \varepsilon(0)=i\right] \\
& =e^{-r T} \int_{0}^{\infty} \int_{0}^{T} \frac{y}{y+K} \phi(\ln (y+K), m(t), v(t)) f_{i}(t, T) d t d y
\end{aligned}
$$

where $\phi(x, m(t), v(t))$ is the normal density function with mean $m(t)$ and variance $v(t)$,

$$
\begin{gathered}
m(t)=\ln \left(X_{0}\right)+\left(r T-\frac{1}{2} v(t)\right), \\
v(t)=\left(\sigma_{0}^{2}-\sigma_{1}^{2}\right) t+\sigma_{1}^{2} T \\
f_{0}(t, T)=e^{-\lambda_{0} T} \delta_{0}(T-t)+e^{-\lambda_{1}(T-t)-\lambda_{0} t}\left[\lambda_{0} I_{0}\left(2\left(\lambda_{0} \lambda_{1} t(T-t)\right)^{1 / 2}\right)\right. \\
\left.+\left(\frac{\lambda_{0} \lambda_{1} t}{T-t}\right)^{1 / 2} I_{1}\left(2\left(\lambda_{0} \lambda_{1} t(T-t)\right)^{1 / 2}\right)\right] \\
f_{1}(t, T)=e^{-\lambda_{1} T} \delta_{0}(t)+e^{-\lambda_{1}(T-t)-\lambda_{0} t}\left[\lambda_{1} I_{0}\left(2\left(\lambda_{0} \lambda_{1} t(T-t)\right)^{1 / 2}\right)\right. \\
\left.+\left(\frac{\lambda_{0} \lambda_{1}(T-t)}{t}\right)^{1 / 2} I_{1}\left(2\left(\lambda_{0} \lambda_{1} t(T-t)\right)^{1 / 2}\right)\right],
\end{gathered}
$$

where $I_{0}$ and $I_{1}$ are the modified Bessel functions, defined as $(a=0,1)$

$$
I_{a}(z)=\left(\frac{z}{2}\right)^{a} \sum_{k=0}^{\infty} \frac{(z / 2)^{2 k}}{k ! \Gamma(k+a+1)},
$$

and $\delta_{0}$ represents unit point mass at 0 .

\section{Remarks:}


a. When $\mu_{0}=\mu_{1}, \sigma_{0}=\sigma_{1}, m(t)$ and $v(t)$ are independent of $t$, and (2.3) reduces to the classical Black-Scholes formula for European call options.

b. When we are not certain about the true state, we can use the stationary distribution $\pi=\left(\pi_{0}, \pi_{1}\right)$ to compute the option price according to $V=$ $\pi_{0} V_{0}+\pi_{1} V_{1}$.

c. Note that we can write (2.3) as a single layer integral with respect to the occupation time probability measure $f_{i}(t, T) d t$ and the integrand equals to the celebrated Black-Scholes formula for European call option with volatility $v(t)$. This representation says that our pricing formula is a mixture of Black-Scholes formula with occupation time distribution as the mixing distribution. This remark also applies to (2.6) when the number of states is larger than 2 .

To gain better understanding of the behavior of the option price under HMM, we compare the option price of a two-state HMM with those computed from two Black-Scholes models. These two Black-Scholes models corresponds to the situation where the HMM stays in states 0 or 1 through out whole time period.

In Figure 1, we use high transition rates $\lambda_{0}=\lambda_{1}=10$ to accelerate mixing.

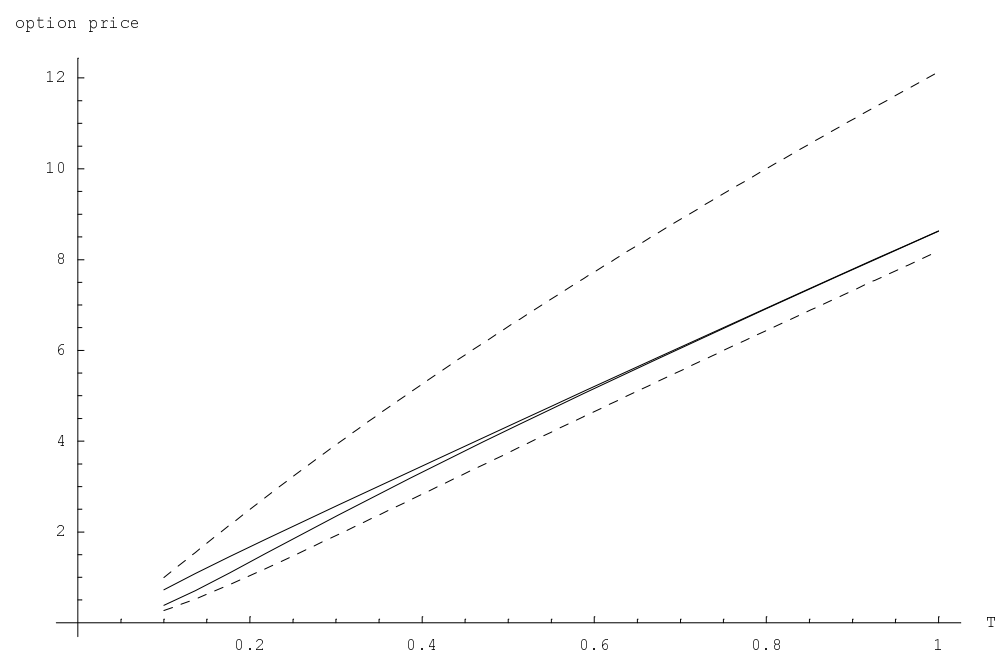

Figure 1: Option prices for the Black-Scholes model and a two-state HMM. Parameter values: $X_{0}=100, K=110, \lambda_{0}=\lambda_{1}=10, r=0.1, \sigma_{0}=0.2, \sigma_{1}=$ 0.3 . The dashed lines represent the option prices given by the classical BlackScholes model for each of the two states, and the solid lines represent the prices given by (2.3) 
It is easy to see that the option price under HMM for short contract duration is close to the corresponding prices given by the Black-Scholes models. Note that the option prices computed from (2.3) are all between those given by the two classical Black-Scholes models, which come together as $T$ increases. This shows that for longer contract duration the initial state becomes less important as the Markov chain settles down in the stationary distribution.

\section{The Case of Finite Number of States}

Next we describe a general approach to option price computation for a finitestate HMM with state space $\{0,1, \cdots, N\}$. Let $\mathbf{t}=\left(t_{0}, t_{1}, \cdots, t_{N}\right)$. To obtain the option prices for a finite-state HMM, we need to evaluate the following integral as in (2.3) for a two-state.

$V_{i}(T, K, r)=e^{-r T} \int_{0}^{\infty} \int_{\left\{\mathbf{t} \mid t_{0}+\cdots+t_{n}=T\right\}} \frac{y}{y+K} \phi\left(\ln (y+K), m(\mathbf{t}), v(\mathbf{t}) f_{i}(\mathbf{t}, T) d \mathbf{t} d y\right.$

where

$$
\begin{aligned}
m(\mathbf{t}) & =\ln \left(X_{0}\right)+\left(r T-\frac{1}{2} v(\mathbf{t})\right), \\
v(\mathbf{t}) & =\sum_{i=0}^{N} \sigma_{i}^{2} t_{i},
\end{aligned}
$$

and $f_{i}(\mathbf{t}, T)$ is the join density of the occupation times, up to time $T$, of the Markov chain $\varepsilon(t)$ starting in state $i$.

In order to evaluate (3.1), we need to find $f_{i}(\mathbf{t}, T), i=0,1, \cdots, N$. In the following, we will demonstrate a method to determine $f_{i}$ with a three-state example. In principle the method can be applied to arbitrary number of states. Note that empirical studies indicate that the number of states rarely go beyond three.

Let $f_{i}\left(t_{0}, t_{1}, T\right)$ be the joint density of occupation times of states 0 and 1 up to time $T$ for the Markov chain $\varepsilon(t)$ starting in state $i$. Note that there is no need to include $t_{2}$ as $t_{2}=T-t_{0}-t_{1}$. To find $f_{i}(u, t, T)$, we can follow the procedure given in the Appendix for a two-state HMM. That is inverting the Laplace transform of $f_{i}$, which satisfies a system of linear equations.

Let $\{\varepsilon(t), t \geq 0\}$ be the underlying continuous time Markov process. Denote the transition probability from state $i$ at time 0 to state $j$ at time $t$ by

$$
p_{t}(i, j)=P(\varepsilon(t)=j \mid \varepsilon(0)=i)
$$


Then

$$
\lambda_{i j}=\lim _{h \rightarrow 0} \frac{p_{h}(i, j)}{h},
$$

defines the jump rates from $i$ to $j(i \neq j)$ and $\lambda_{i}=\sum_{j \neq i} \lambda_{i j}$ is the rate of transitions out of state $i$. Consider the following transition rate matrix for the three-state Markov chain

$$
\left[\begin{array}{lll}
-\lambda_{0} & \lambda_{0} & 0 \\
\lambda_{10} & -\lambda_{1} & \lambda_{12} \\
0 & \lambda_{2} & -\lambda_{2}
\end{array}\right],
$$

where $\lambda_{10}+\lambda_{12}=\lambda_{1}$. The preceding transition rate matrix implies that the Markov chain must go through the transitional state $\varepsilon(t)=1$ when moving between state 0 and state 2 . Our method can handle chains with general transition rate matrices and we use this plausible case to facilitate our presentation. This is a difference between two-state model and three-state model.

Applying the same argument as (A.2) in the Appendix 1, we find that the Laplace transformation $\psi_{i}$ of $f_{i} i=0,1,2$ satisfies the following system of linear equations

$$
\begin{aligned}
& \hat{\psi}_{0}(r, s, t)=\frac{1}{r+t+\lambda_{0}}\left[1+\lambda_{0} \hat{\psi}_{1}(r, s, t)\right], \\
& \hat{\psi}_{1}(r, s, t)=\frac{1}{s+t+\lambda_{1}}\left[1+\lambda_{10} \hat{\psi}_{0}(r, s, t)+\lambda_{12} \hat{\psi}_{2}(r, s, t)\right], \\
& \hat{\psi}_{2}(r, s, t)=\frac{1}{t+\lambda_{2}}\left[1+\lambda_{2} \hat{\psi}_{1}(r, s, t)\right] .
\end{aligned}
$$

Solving the preceding equations, we obtain

$$
\begin{aligned}
& \hat{\psi}_{0}(r, s, t)=\left[t+\frac{r \phi(s, t)+\lambda_{0}\left(\lambda_{2}+t\right) s}{\phi(s, t)+\lambda_{0}\left(\lambda_{2}+t+\lambda_{1}-\lambda_{10}\right)}\right]^{-1}, \\
& \hat{\psi}_{1}(r, s, t)=\left[t+\frac{s\left(t+\lambda_{2}\right)\left(r+t+\lambda_{0}\right)+r \lambda_{10}\left(t+\lambda_{2}\right)}{\left(t+\lambda_{1}+\lambda_{2}\right)\left(r+t+\lambda_{0}\right)-\lambda_{10}\left(r-\lambda_{2}+\lambda_{0}\right)}\right]^{-1}, \\
& \hat{\psi}_{2}(r, s, t)=\left[t+\frac{\lambda_{2} s\left(r+t+\lambda_{0}\right)+r \lambda_{10} \lambda_{2}}{\left(r+t+\lambda_{0}\right)\left(\lambda_{2}+\lambda_{1}+s+t\right)+\lambda_{10}\left(\lambda_{2}-\lambda_{0}\right)}\right]^{-1},
\end{aligned}
$$

where $\phi(s, t)=\left(s t+\lambda_{2} t+\lambda_{1} t+t^{2}+s \lambda_{2}+\lambda_{2} \lambda_{10}\right)$.

We need to perform inverse Laplace transform on $\hat{\psi}_{i}(r, s, t)$ for each of the three variables in sequence. The result is given in the Appendix 2.

When the number of states goes beyond three, the inverse Laplace transformation may be difficult to carry out analytically. In this case, we can solve 
numerically. The problem of two-state model is solved analytically, the threestate or more can be solved the problem numerically. The strategy is to invert the Laplace transform analytically for some variables and then perform numerical inversion of Laplace transform for the rest. One way to do numerical inversion of Laplace transformation is through the Fourier series expansion algorithm given by (Choudhury, Lucantoni and Whitt, 1994).

\section{Numerical Performance}

\subsection{Design of the Simulation}

Here we compared European call option prices obtained using discrete diffusion method and the Markovian tree method and the closed form formula (3.1). Two-state and three-state hidden Markov models were considered in the simulation study, respectively. We used the exact analytic formula as the benchmark for both the two-state and three-state HMM. The results shown in Tables 1 to 10 depend on four factors:

a. Low and high volatilities;

b. Transition rates for the three-state HMM;

c. Strike prices: in the money, at the money and out of the money;

d. Expiration dates: $T=0.1,0.2,0.5,1,2,3$ years.

Since model (2.1) is a continuous time model, we discretize this continuous time model following (Cox, Ross and Rubinstein, 1979). A corresponding scheme for a two-state HMM can be found in (David, 1997). It is worth pointing out that this method can also be applied to the general case, where the hidden Markov process $\varepsilon(t)$ has more than three states. That is, the state space is $\Omega=\{0,1, \cdots, N\}$, where more complex information patterns can be described.

Let $\eta$ be the standard normal random variable $N(0,1)$. We rewrite $(2.2)$ in the discrete time form

$$
X_{n+h}=X_{n} e^{\left(r-\sigma_{\varepsilon_{n}}^{2} / 2\right) h+\sigma_{\varepsilon_{n}} \sqrt{h} \eta},
$$

where $\varepsilon_{n}$ is the corresponding discrete time Markov chain with probability of changing state $\left(\delta_{i j}+(-1)^{\delta_{i j}} e^{-\lambda_{i} h}\right)\left(\lambda_{i j} / \lambda_{i}\right)$ with $\lambda_{i i}=\lambda_{i}, \delta_{i i}=0, \delta_{i j}=1, i \neq j$. We repeat the steps $M$ times, where $M$ is sufficiently large to guarantee convergence of the simulation.

To illustrate the idea of the Markovian tree method, we divide the time interval $[0, t]$ into $n$ sub-intervals such that $t=n h$. Let $X=\left(X_{k}, k \geq 0\right)$, and let 
$X_{k}$ be a price at time $k h$. Define

$$
X_{k}^{\varepsilon_{k}}=\left(X_{k}, \varepsilon_{k}\right)=(X(k h), \varepsilon(k h)) .
$$

Let $\eta_{n}^{i, j}$ be independent and identically distributed (i.i.d.) random variables, taking the values $u_{j}$ with probability $p_{j}\left(\delta_{i, j}+(-1)^{\delta_{i, j}} e^{-\lambda_{i} h}\right)\left(\lambda_{i j} / \lambda_{i}\right)$ and $1 / u_{j}$ with probability $\left(1-p_{j}\right)\left(\delta_{i, j}+(-1)^{\delta_{i, j}} e^{-\lambda_{i} h}\right)\left(\lambda_{i j} / \lambda_{i}\right), i, j=0,1, \cdots, N$, respectively), where

$$
u_{i}=e^{\sigma_{i} \sqrt{h}}, \quad p_{i}=\frac{\mu_{i} h+\sigma_{i} \sqrt{h}-0.5 \sigma_{i}^{2} h}{2 \sigma_{i} \sqrt{h}} .
$$

The $p_{i}$ can be found by comparing the Fourier transformation $E\left(e^{i c Y_{t}}\right)$ of the continuous process $Y_{t}=\log X_{t}$ and that of the discrete process $Y_{k}^{\varepsilon_{k}}=Y_{k-1}^{\varepsilon_{k-1}} \pm$ $\sigma_{j} \sqrt{h}, j=0,1, \cdots, N$, so that the later converges to the same system of ordinary differential equations by the former.

We have the following recurrence relation:

$$
X_{n}=\eta_{n}^{\varepsilon(n), \varepsilon(n-1)} X_{n-1} .
$$

By the memoryless property of $\tau_{i},\left(X_{n}^{\varepsilon_{n}}, n \geq 0\right)$ is a Markov chain. The Markov chain $X_{n}^{\varepsilon_{n}}$ with initial state $X_{0}=x$ is a random walk on the set

$$
E_{x}=\left\{x u^{r} \mid r=\sigma_{0} n_{0}+\sigma_{1} n_{1}+\cdots+\sigma_{N} n_{N}, n_{0}, n_{1}, \cdots, n_{N} \in Z, u=e^{\sqrt{h}}\right\} .
$$

Here we took $n$, the number of sub-intervals, as 30 since our simulation showed that this number is large enough to provide accurate results. The Monte Carlo replication size for both discrete diffusion method and the Markovian tree method is $B=5,000,000$. Computations were performed using Visual Basic programs on a personal computer system with a Pentium $4 \mathrm{CPU}, 1.6 \mathrm{G}$ and $256 \mathrm{MB}$ of RAM, at the Institute of Statistical Science, Academia Sinica, Taipei, Taiwan, R.O.C.. The pseudo-random numbers were generated by using IMSL routines. The reported running time is the CPU time in seconds.

\subsection{Simulation Results}

We use the following notations in Tables 1 to 10. B-S $i$ refers to the classical Black-Scholes formula for states $i=0,1,2 ; V_{i}$ the exact price based on (3.1); dis $i$ and tree $i$ discrete diffusion and the Markovian tree method, respectively.

We will first consider the case of a two-state HMM with same transition rates. Tables 1 and 2 report the numerical and simulation results according to low and high volatilities, respectively. In general, the results produced by the discrete diffusion method and the Markovian tree method are very close for various expiration dates and both are better for shorter period. It is particularly 
interesting to observe that discrete diffusion gives better results than the Markov tree method. In terms of computing time, the closed form formula requires about $1 / 100$ of what it takes for the Markovian tree, which in turns takes about $1 / 2$ of what requires of discrete diffusion.

Table 1: Option prices for a two-state HMM (low volatility) $X_{0}=100, K=$ $100, \lambda_{0}=\lambda_{1}=1, r=0.1, \sigma_{0}=0.2, \sigma_{1}=0.3, n=30$

\begin{tabular}{ccccccccc}
\hline$T$ (year) & B-S 0 & B-S 1 & $V_{0}$ & $V_{1}$ & dis 0 & dis 1 & tree 0 & tree 1 \\
\hline 0.1 & 3.038 & 4.282 & 3.101 & 4.228 & 3.096 & 4.228 & 3.078 & 4.176 \\
0.2 & 4.609 & 6.344 & 4.772 & 6.201 & 4.765 & 6.200 & 4.764 & 6.144 \\
0.5 & 8.277 & 10.906 & 8.793 & 10.454 & 8.780 & 10.454 & 8.770 & 10.466 \\
1.0 & 13.269 & 16.734 & 14.317 & 15.813 & 14.291 & 15.832 & 14.266 & 15.730 \\
2.0 & 21.719 & 25.975 & 23.418 & 24.461 & 23.382 & 24.474 & 23.336 & 24.472 \\
3.0 & 29.074 & 33.604 & 31.053 & 31.806 & 31.042 & 31.806 & 30.856 & 31.633 \\
\hline run time(sec) & $<10^{-5}$ & $<10^{-5}$ & 1.51 & 1.45 & 240.55 & 251.32 & 140.24 & 144.23 \\
\hline
\end{tabular}

This table reports the European call options for a two-state HMM with low and high volatilities. B-S $i$ refers to the classical Black-Scholes formula for states $i=0,1 ; V_{i}$ the exact price based on (3.1); dis $i$ and tree $i$ discrete diffusion and the Markovian tree method, respectively.

Table 2: Option prices for a two-state HMM (high volatility) $X_{0}=100, K=$ $100, \lambda_{0}=\lambda_{1}=1, r=0.1, \sigma_{0}=0.1, \sigma_{1}=1.0, n=30$

\begin{tabular}{ccccccccc}
\hline$T$ (year) & B-S 0 & B-S 1 & $V_{0}$ & $V_{1}$ & dis 0 & dis 1 & tree 0 & tree 1 \\
\hline 0.1 & 1.814 & 13.004 & 2.516 & 12.624 & 2.467 & 12.647 & 2.475 & 12.553 \\
0.2 & 2.930 & 18.525 & 4.694 & 17.533 & 4.651 & 17.595 & 4.649 & 17.443 \\
0.5 & 5.850 & 29.461 & 11.445 & 26.343 & 11.381 & 26.444 & 11.456 & 26.263 \\
1.0 & 10.308 & 41.395 & 21.939 & 35.143 & 21.805 & 35.272 & 21.839 & 35.191 \\
2.0 & 18.580 & 56.793 & 37.913 & 46.841 & 37.786 & 47.155 & 37.849 & 46.850 \\
3.0 & 26.169 & 66.973 & 49.205 & 55.510 & 49.187 & 55.724 & 49.038 & 55.413 \\
\hline run time(sec) & $<10^{-5}$ & $<10^{-5}$ & 1.44 & 1.52 & 249.46 & 252.55 & 100.23 & 101.17 \\
\hline
\end{tabular}

This table reports the European call options for a two-state HMM with high volatilities. B-S $i$ refers to the classical Black-Scholes formula for states $i=0,1 ; V_{i}$ the exact price based on (3.1); dis $i$ and tree $i$ discrete diffusion and the Markovian tree method, respectively.

Let $Q_{1}$ be the matrix of transition rate

$$
Q_{1}=\left[\begin{array}{lll}
-1 & 1 & 0 \\
1 / 2 & -1 & 1 / 2 \\
0 & 1 & -1
\end{array}\right]
$$

and let $Q_{2}$ be the matrix of transition rate 


$$
Q_{2}=\left[\begin{array}{lll}
-1 & 1 & 0 \\
5 / 2 & -5 & 5 / 2 \\
0 & 10 & -10
\end{array}\right] .
$$

The results for a three-state HMM model are reported in Tables 3 to 6 . Note that $Q_{1}$ represents a Markov chain with similar transition rates out of each state, while $Q_{2}$ represents a chain with very different transition rates.

Tables 3 and 4 display the option prices for a three-state HMM with the transition rate $Q_{1}$ and with low and high volatility, respectively. Tables 5 and 6 contain option prices for a HMM with the transition rate matrix $Q_{2}$ and low and high volatility, respectively.

Table 3: Option prices for a three-state HMM with the transition rate $Q_{1}$ (low volatility) $X_{0}=100, K=100, \lambda_{0}=\lambda_{1}=\lambda_{2}=1, r=0.1, \sigma_{0}=0.1, \sigma_{1}=$ $0.2, \sigma_{2}=0.3, n=30$

\begin{tabular}{cccccccccc}
\hline$T$ (year) & dis 0 & dis 1 & dis 2 & tree 0 & tree 1 & tree 2 & $V_{0}$ & $V_{1}$ & $V_{2}$ \\
\hline 0.1 & 1.876 & 3.034 & 4.231 & 1.884 & 3.016 & 4.182 & 1.898 & 3.346 & 4.309 \\
0.2 & 3.096 & 4.607 & 6.207 & 3.078 & 4.619 & 6.141 & 3.206 & 4.886 & 6.094 \\
0.5 & 6.390 & 8.271 & 10.443 & 6.352 & 8.226 & 10.450 & 6.572 & 8.721 & 10.350 \\
1.0 & 11.408 & 13.266 & 15.698 & 11.344 & 13.139 & 15.507 & 11.461 & 13.761 & 15.444 \\
2.0 & 20.302 & 21.712 & 23.971 & 20.285 & 21.293 & 23.964 & 20.201 & 21.736 & 23.776 \\
3.0 & 28.052 & 29.047 & 30.961 & 27.858 & 28.866 & 30.729 & 27.333 & 28.426 & 30.386 \\
\hline run time(sec) & 269.25 & 272.84 & 276.63 & 171.25 & 175.22 & 178.32 & 2.49 & 2.71 & 2.49 \\
\hline
\end{tabular}

This table reports the European call options for a three-state HMM with low volatility and $Q_{1} . V_{i}$ the exact price based on (3.1); dis $i$ and tree $i$ discrete diffusion and the Markovian tree method, respectively.

Table 4: Option prices for a three-state HMM with the transition rate $Q_{1}$ (high volatility) $X_{0}=100, K=100, \lambda_{0}=\lambda_{1}=\lambda_{2}=1, r=0.1, \sigma_{0}=0.8, \sigma_{1}=$ $0.9, \sigma_{2}=1.0, n=30$

\begin{tabular}{cccccccccc}
\hline$T$ (year) & dis 0 & dis 1 & dis 2 & tree 0 & tree 1 & tree 2 & $V_{0}$ & $V_{1}$ & $V_{2}$ \\
\hline 0.1 & 10.578 & 11.763 & 12.933 & 10.498 & 11.726 & 12.859 & 10.584 & 11.799 & 12.821 \\
0.2 & 15.217 & 16.806 & 18.373 & 15.074 & 16.718 & 18.260 & 15.170 & 16.758 & 18.262 \\
0.5 & 24.782 & 26.884 & 28.974 & 24.494 & 26.675 & 28.609 & 24.518 & 26.602 & 28.606 \\
1.0 & 35.787 & 37.990 & 40.258 & 35.732 & 37.776 & 39.989 & 35.764 & 37.755 & 39.974 \\
2.0 & 50.881 & 52.853 & 54.706 & 50.564 & 51.955 & 54.095 & 50.860 & 51.945 & 54.002 \\
3.0 & 61.332 & 62.665 & 64.456 & 60.727 & 61.948 & 63.907 & 60.782 & 61.982 & 63.994 \\
\hline run time(sec) & 267.11 & 282.29 & 288.13 & 164.54 & 170.91 & 178.30 & 2.47 & 2.70 & 2.48 \\
\hline
\end{tabular}

This table reports the European call options for a three-state HMM with high volatility and $Q_{1} . V_{i}$ the exact price based on (3.1); dis $i$ and tree $i$ discrete diffusion and the Markovian tree method, respectively. 
496 Cheng-Der Fuh, Kwok Wah Remus Ho, Inchi Hu and Ren-Her Wang

Table 5: Option prices for a three-state HMM with the transition rate $Q_{2}$ (low volatility) $X_{0}=100, K=100, \lambda_{0}=1, \lambda_{1}=5, \lambda_{2}=10, r=0.1, \sigma_{0}=$ $0.1, \sigma_{1}=0.2, \sigma_{2}=0.3, n=30$

\begin{tabular}{cccccccccc}
\hline$T$ (year) & dis 0 & dis 1 & dis 2 & tree 0 & tree 1 & tree 2 & $V_{0}$ & $V_{1}$ & $V_{2}$ \\
\hline 0.1 & 1.877 & 3.037 & 3.863 & 1.882 & 3.014 & 3.841 & 1.891 & 3.089 & 3.827 \\
0.2 & 3.093 & 4.610 & 5.383 & 3.078 & 4.546 & 5.400 & 3.073 & 4.581 & 5.471 \\
0.5 & 6.388 & 8.276 & 8.675 & 6.318 & 7.958 & 8.769 & 6.332 & 7.957 & 8.760 \\
1.0 & 11.420 & 13.260 & 13.343 & 11.159 & 12.484 & 13.153 & 11.173 & 12.499 & 13.182 \\
2.0 & 20.321 & 21.711 & 21.632 & 19.755 & 20.570 & 21.032 & 19.742 & 20.590 & 21.071 \\
3.0 & 28.050 & 29.038 & 28.970 & 27.192 & 27.765 & 28.115 & 27.145 & 27.788 & 28.159 \\
\hline run time(sec) & 263.01 & 264.90 & 263.45 & 172.78 & 171.44 & 174.20 & 2.51 & 2.70 & 2.51 \\
\hline
\end{tabular}

This table reports the European call options for a three-state HMM with low volatility and $Q_{2} . V_{i}$ the exact price based on (3.1); dis $i$ and tree $i$ discrete diffusion and the Markovian tree method, respectively.

Table 6: Option prices for a three-state HMM with the transition rate $Q_{2}$ (high volatility) $X_{0}=100, K=100, \lambda_{0}=1, \lambda_{1}=5, \lambda_{2}=10, r=0.1, \sigma_{0}=$ $0.8, \sigma_{1}=0.9, \sigma_{2}=1.0, n=30$

\begin{tabular}{cccccccccc}
\hline$T$ (year) & dis 0 & dis 1 & dis 2 & tree 0 & tree 1 & tree 2 & $V_{0}$ & $V_{1}$ & $V_{2}$ \\
\hline 0.1 & 10.581 & 11.760 & 12.549 & 10.486 & 11.678 & 12.494 & 10.490 & 11.667 & 12.467 \\
0.2 & 15.225 & 16.794 & 17.495 & 15.064 & 16.617 & 17.506 & 15.099 & 16.623 & 17.523 \\
0.5 & 24.800 & 26.882 & 27.119 & 24.403 & 26.279 & 27.069 & 24.414 & 26.227 & 27.027 \\
1.0 & 35.850 & 38.047 & 37.870 & 35.419 & 36.866 & 37.508 & 35.407 & 36.843 & 37.543 \\
2.0 & 50.900 & 52.778 & 52.502 & 49.881 & 50.811 & 51.343 & 49.893 & 50.811 & 51.311 \\
3.0 & 61.550 & 62.751 & 62.509 & 59.739 & 60.420 & 60.596 & 59.705 & 60.469 & 60.469 \\
\hline run time(sec) & 261.34 & 265.69 & 267.45 & 171.34 & 182.22 & 183.20 & 2.52 & 2.73 & 2.59 \\
\hline
\end{tabular}

This table reports the European call options for a three-state HMM with high volatility and $Q_{2} . V_{i}$ the exact price based on (3.1); dis $i$ and tree $i$ discrete diffusion and the Markovian tree method, respectively.

Examination of Tables 3 to 6 , we obtain the following conclusions. In general, the discrete diffusion method is better than the Markovian tree method when the transition rates are similar $\left(Q_{1}\right)$. When the transition rates are very different $\left(Q_{2}\right)$, the Markov tree method generally gives better results. Secondly, when the volatility are low, the option prices for different initial states are closer to each other and are lower than the corresponding option prices under high volatility. Thirdly, in Tables 5 and 6 the option prices are close to each other because states 1 and 2 have high transition rates $\left(\lambda_{1}=5, \lambda_{2}=10\right)$, and because most of the time the chain stays in state 0 . Furthermore, the prices starting in states 1 and 2 are closer than those starting in state 0 . In other words, option prices with many initial states will merge into a few, if the chain spends most of the time in a few 
states. In this case, one does not need many states to model stock returns from option price computation point of view.

To show the effect of strike prices, in Tables 7 and 8, we provide European call option prices for various $K / X_{0}$ values in a hidden Markov model with three

Table 7: Difference in option prices for a three-state HMM with rate matrix $Q_{2}$ (low volatility) $X_{0}=100, \lambda_{0}=1, \lambda_{1}=5, \lambda_{2}=10, r=0.1, \sigma_{0}=0.1, \sigma_{1}=$ $0.2, \sigma_{2}=0.3, n=30$

\begin{tabular}{|c|c|c|c|c|c|c|}
\hline \multirow[b]{2}{*}{$K / X_{0}$} & \multicolumn{3}{|c|}{$T=0.1$} & \multicolumn{3}{|c|}{$T=0.2$} \\
\hline & 1.1 & 1.0 & 0.9 & 1.1 & 1.0 & 0.9 \\
\hline$V_{0}$ & 0.0146 & 1.8805 & 10.899 & 0.1753 & 3.1001 & 11.810 \\
\hline tree 0 & 0.0128 & 1.8829 & 10.906 & 0.1641 & 3.0783 & 11.845 \\
\hline difference & -0.1233 & 0.0013 & 0.0007 & -0.0639 & -0.0070 & 0.0030 \\
\hline Std & 0.0001 & 0.0010 & 0.0016 & 0.0004 & 0.0016 & 0.0022 \\
\hline$V_{1}$ & 0.2928 & 3.0304 & 10.993 & 1.0226 & 4.5389 & 12.112 \\
\hline tree 1 & 0.2884 & 3.0140 & 10.925 & 1.0173 & 4.5460 & 12.178 \\
\hline difference & -0.0150 & -0.0054 & -0.0062 & -0.0052 & 0.0016 & 0.0054 \\
\hline Std & 0.0006 & 0.0018 & 0.0028 & 0.0013 & 0.0027 & 0.0036 \\
\hline$V_{2}$ & 0.7309 & 3.8535 & 11.227 & 1.6897 & 5.3945 & 12.471 \\
\hline tree 2 & 0.7362 & 3.8411 & 11.191 & 1.7075 & 5.4005 & 12.473 \\
\hline difference & 0.0073 & -0.0032 & -0.0032 & 0.0105 & 0.0011 & 0.0002 \\
\hline \multirow[t]{2}{*}{ Std } & 0.0011 & 0.0025 & 0.0035 & 0.0020 & 0.0034 & 0.0045 \\
\hline & \multicolumn{3}{|c|}{$T=0.5$} & \multicolumn{3}{|c|}{$T=1.0$} \\
\hline$K / X_{0}$ & 1.1 & 1.0 & 0.9 & 1.1 & 1.0 & 0.9 \\
\hline$V_{0}$ & 1.6835 & 6.3548 & 14.577 & 5.4750 & 11.206 & 18.991 \\
\hline tree 0 & 1.6462 & 6.3186 & 14.636 & 5.4421 & 11.159 & 18.904 \\
\hline difference & -0.0222 & -0.0057 & 0.0040 & -0.0060 & -0.0042 & -0.0046 \\
\hline Std & 0.0018 & 0.0031 & 0.0037 & 0.0040 & 0.0051 & 0.0058 \\
\hline$V_{1}$ & 3.3243 & 7.8874 & 15.171 & 7.0659 & 12.406 & 19.565 \\
\hline tree 1 & 3.3714 & 7.9582 & 15.187 & 7.1656 & 12.484 & 19.508 \\
\hline difference & 0.0142 & 0.0090 & 0.0011 & 0.0141 & 0.0063 & -0.0029 \\
\hline Std & 0.0031 & 0.0044 & 0.0054 & 0.0053 & 0.0065 & 0.0074 \\
\hline$V_{2}$ & 4.1288 & 8.6440 & 15.560 & 7.7930 & 12.983 & 19.882 \\
\hline tree 2 & 4.2281 & 8.7693 & 15.632 & 8.0111 & 13.153 & 19.873 \\
\hline difference & 0.0241 & 0.0145 & 0.0046 & 0.0280 & 0.0131 & -0.0004 \\
\hline Std & 0.0038 & 0.0051 & 0.0061 & 0.0059 & 0.0071 & 0.0081 \\
\hline
\end{tabular}

This table reports the European call options for a three-state HMM with low volatility and three different strike-to-stock price ratios $K / X_{0}$. They were $1.1,1.0$ and 0.9 , which correspond to out of the money, at the money, and in the money cases, respectively. 
Table 8: Difference in option prices for a three-state HMM with rate matrix $Q_{2}$ (high volatility) $X_{0}=100, \lambda_{0}=1, \lambda_{1}=5, \lambda_{2}=10, r=0.1, \sigma_{0}=0.8, \sigma_{1}=$ $0.9, \sigma_{2}=1.0, n=30$

\begin{tabular}{|c|c|c|c|c|c|c|}
\hline \multirow[b]{2}{*}{$K / X_{0}$} & \multicolumn{3}{|c|}{$T=0.1$} & \multicolumn{3}{|c|}{$T=0.2$} \\
\hline & 1.1 & 1.0 & 0.9 & 1.1 & 1.0 & 0.9 \\
\hline$V_{0}$ & 6.7102 & 10.582 & 15.984 & 11.327 & 15.228 & 20.197 \\
\hline tree 0 & 6.6165 & 10.486 & 15.689 & 11.408 & 15.064 & 20.111 \\
\hline difference & -0.0140 & -0.0090 & -0.0184 & 0.0071 & -0.0108 & -0.0043 \\
\hline Std & 0.0064 & 0.0079 & 0.0094 & 0.0106 & 0.0121 & 0.0134 \\
\hline$V_{1}$ & 7.8632 & 11.736 & 16.984 & 12.825 & 16.682 & 21.506 \\
\hline tree 1 & 7.7310 & 11.678 & 16.790 & 12.898 & 16.617 & 21.599 \\
\hline difference & -0.0168 & -0.0050 & -0.0114 & 0.0057 & -0.0039 & 0.0043 \\
\hline Std & 0.0075 & 0.0089 & 0.0103 & 0.0122 & 0.0136 & 0.0148 \\
\hline$V_{2}$ & 8.6818 & 12.5500 & 17.610 & 13.687 & 17.517 & 22.263 \\
\hline tree 2 & 8.6757 & 12.494 & 17.653 & 13.824 & 17.506 & 22.366 \\
\hline difference & -0.0007 & -0.0045 & -0.0026 & 0.0100 & -0.0006 & 0.0046 \\
\hline \multirow[t]{2}{*}{ Std } & 0.0083 & 0.0097 & 0.0110 & 0.0131 & 0.0145 & 0.0157 \\
\hline & \multicolumn{3}{|c|}{$T=0.5$} & \multicolumn{3}{|c|}{$T=1.0$} \\
\hline$K / X_{0}$ & 1.1 & 1.0 & 0.9 & 1.1 & 1.0 & 0.9 \\
\hline$V_{0}$ & 21.056 & 24.740 & 29.067 & 32.246 & 35.529 & 39.231 \\
\hline tree 0 & 21.188 & 24.403 & 28.953 & 32.467 & 35.419 & 39.541 \\
\hline difference & 0.0063 & -0.0136 & -0.0039 & 0.0069 & -0.0031 & 0.0079 \\
\hline Std & 0.0209 & 0.0222 & 0.0235 & 0.0366 & 0.0377 & 0.0388 \\
\hline$V_{1}$ & 22.723 & 26.328 & 30.530 & 33.632 & 36.841 & 40.449 \\
\hline tree 1 & 22.944 & 26.279 & 30.714 & 33.856 & 36.866 & 40.607 \\
\hline difference & 0.0097 & -0.0019 & 0.0060 & 0.0067 & 0.0007 & 0.0039 \\
\hline Std & 0.0233 & 0.0246 & 0.0258 & 0.0397 & 0.0408 & 0.0418 \\
\hline$V_{2}$ & 23.478 & 27.048 & 31.194 & 34.213 & 37.391 & 40.959 \\
\hline tree 2 & 23.687 & 27.069 & 31.353 & 34.632 & 37.508 & 41.218 \\
\hline difference & 0.0089 & 0.0008 & 0.0051 & 0.0123 & 0.0031 & 0.0063 \\
\hline Std & 0.0244 & 0.0257 & 0.0269 & 0.0411 & 0.0421 & 0.0432 \\
\hline
\end{tabular}

This table reports the European call options for a three-state HMM with high volatility and three different strike-to-stock price ratios $K / X_{0}$. They were $1.1,1.0$ and 0.9 , which correspond to out of the money, at the money, and in the money cases, respectively.

states. For initial stock price $X_{0}$, we considered three different strike-to-stock price ratios $K / X_{0}$. They were $1.1,1.0$ and 0.9 , which correspond to out of the money, at the money, and in the money cases, respectively. Note that in Tables 7 and 8, the first row in each panel lists the prices based on analytical formula with different initial states, whereas the second and fourth rows list Markov tree 
prices and their standard deviations, respectively. The reason why we pick the Markov tree instead of the discrete diffusion is because in Tables 3-6 we have observed that the former performs better under $Q_{2}$. The numbers in the third row are the error estimates between the exact price and the results of Markov tree given by "relative difference"

$$
\text { rel. diff. }=\frac{\text { tree } \mathrm{i}-V_{i}}{V_{i}} .
$$

In Table 7, where the volatility are low, the Markov tree method is best for in-the-money case, second best for at-the-money case, and worst for out-ofthe-money case. In Table 8, high volatility case, the tree method is best for at-the-money case, in-the-money case comes in second, and out-of-the-money is worst.

\subsection{Sensitivity Analysis}

The option price depends on several parameters, $\sigma, \lambda$ etc. in a complicated way as shown by (2.3) and (3.1). It would be helpful to know the impact of each parameter on the option price. Here we carry out a study of their influence. Tables 9 and 10 show the sensitivity of the parameters to option prices for a two-state and a three-state HMM, respectively. The values listed in the table are the values of the stock price with small perturbation, a $10 \%$ increase, for the indicated parameter while all other parameters remain fixed.

The preceding results show that the volatility $\sigma$ has the more significant effect than $\lambda$. We also note that the increase of $\sigma$ 's in different states has almost the same effect on the option price.

Table 9: Sensitivity analysis for a two-state HMM Base parameters values:

$X_{0}=100, K=100, T=1, \lambda_{0}=1, \lambda_{1}=10, \quad r=0.06, \sigma_{0}=0.05, \sigma_{1}=0.1$

\begin{tabular}{cccccc}
\hline Valuation & $\begin{array}{c}\text { Perturbed } \\
\text { parameter }\end{array}$ & $V_{0}$ & $V_{1}$ & $\begin{array}{c}\text { Difference } \\
\text { of } V_{0}\end{array}$ & $\begin{array}{r}\text { Difference } \\
\text { of } V_{1}\end{array}$ \\
\hline Base valuation & & 5.5034 & 5.7989 & & \\
$\sigma_{0}$ & 0.055 & 5.6118 & 5.8927 & 0.1084 & 0.0938 \\
$\sigma_{1}$ & 0.11 & 5.5394 & 5.8726 & 0.0360 & 0.0737 \\
$\lambda_{0}$ & 1.1 & 5.5280 & 5.8203 & 0.0246 & 0.0214 \\
$\lambda_{1}$ & 11 & 5.4834 & 5.7553 & -0.0200 & -0.0430 \\
\hline
\end{tabular}

This table reports the sensitivity of the parameters to option prices for a two-state HMM.

\subsection{Volatility Smiles and Surfaces}

If the Black-Scholes model is correct, then the implied volatility should be 
500 Cheng-Der Fuh, Kwok Wah Remus Ho, Inchi Hu and Ren-Her Wang

Table 10: Sensitivity analysis for a three-state HMM $X_{0}=100, K=100, T=$ $1, \lambda_{0}=1, \lambda_{1}=5, \lambda_{2}=10, r=0.06, \sigma_{0}=0.05, \sigma_{1}=0.1, \sigma_{2}=0.2$

\begin{tabular}{|c|c|c|c|c|c|c|c|}
\hline Valuation & $\begin{array}{l}\text { Perturbed } \\
\text { parameter }\end{array}$ & $V_{0}$ & $V_{1}$ & $V_{2}$ & $\begin{array}{l}\text { Difference } \\
\text { of } V_{0}\end{array}$ & $\begin{array}{c}\text { Difference } \\
\text { of } V_{1}\end{array}$ & $\begin{array}{l}\text { Difference } \\
\text { of } V_{2}\end{array}$ \\
\hline Base valuation & & 6.1919 & 7.4978 & 8.2333 & & & \\
\hline$\sigma_{0}$ & 0.055 & 6.2795 & 7.5443 & 8.2692 & 0.0876 & 0.0465 & 0.0359 \\
\hline$\sigma_{1}$ & 0.11 & 6.2576 & 7.6388 & 8.3539 & 0.0657 & 0.1410 & 0.1206 \\
\hline$\sigma_{2}$ & 0.22 & 6.2394 & 7.6086 & 8.4427 & 0.0475 & 0.1108 & 0.2094 \\
\hline$\lambda_{0}$ & 1.1 & 6.2665 & 7.5342 & 8.2614 & 0.0746 & 0.0364 & 0.0281 \\
\hline$\lambda_{1}$ & 5.5 & 6.1818 & 7.4616 & 8.2038 & -0.0101 & -0.0362 & -0.0295 \\
\hline$\lambda_{2}$ & 11 & 6.1722 & 7.4443 & 8.1248 & -0.0197 & -0.0535 & -0.1085 \\
\hline
\end{tabular}

This table reports the sensitivity of the parameters to option prices for a three-state HMM.

constant. However, it is widely recognized that the volatility has a "smile" feature. That is, most derivative markets exhibit persistent patterns of volatility variation by strike. In some markets, those patterns form a smile. (Hull, 2003) pointed out that most empirical results of the implied volatility smile become skew after market crash in 1987. In others, such as equity index options markets, it is more of a skewed curve. This has motivated the term "volatility skew". In practice, either the term volatility smile or volatility skew may be used to refer to the general phenomena of volatilities varying by strike. Model (2.1) can generate the phenomena of the volatility skew, as shown in Figure 2.

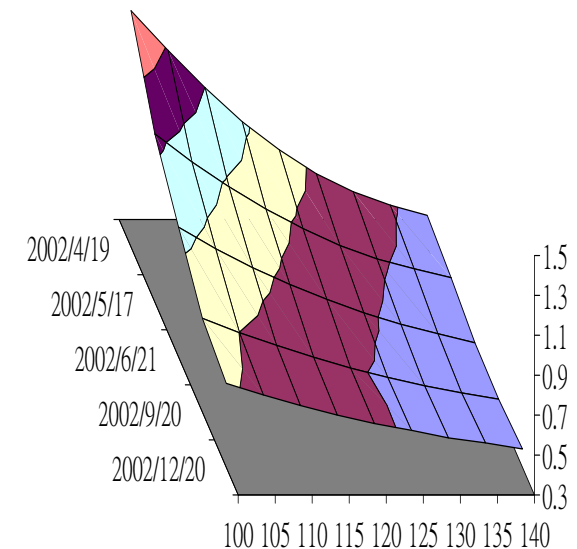

Figure 2: Implied volatilities against expiry and strike price. The parameter values: $X_{0}=107, \lambda_{0}=\lambda_{1}=1, r=0.0261$

We show implied volatility against both maturity and strike in a three-dimensional plot. That is, we can consider $\sigma(X, t)$ as a function of $X$ and $t$. Figure 2 is based on price data of European call option on IBM stock with five different maturity days. The call options were traded on 3/15/2002 with strikes of 100 , 
$105,110,115,120,125,130,135$ and 140 . We use as the riskless rate $r=0.0261$ the yield of 1 -year US TREASURY Bills to maturity on 3/15/2003 and the IBM stock price is 107 on $3 / 15 / 2002$.

This implied surface represents the constant value of volatility that gives each traded option a theoretical value equal to the market value. The time dependence in implied volatility can be viewed as the evidence for the time dependence of volatility of the underlying asset. For $\sigma(X, t)$ deduced from the volatility surface at a specific time $t^{*}$, we might call it the local volatility surface. This local volatility surface can be thought of as the market's view of the future value of volatility when the asset price is $X$ at time $t$.

We should emphasize that the results presented in Figure 2 do not represent an empirical test of the model (2.1); it only illustrates that the model can produce a close fit to the empirical phenomenon.

\section{Conclusions}

The Markov switching model is better in capturing the empirical phenomena of stock prices fluctuation. In this paper, a closed form option pricing formula for the Black-Scholes model with the Markov switching has been developed. Numerical evaluation of the formula was performed for both two-state and three-state hidden Markov models. The close form formula was used as a benchmark to investigate the performance of simulation methods such as the discrete diffusion and the Markovian tree methods. The pricing error is given in terms of relative difference the exact price produced by the analytical formula.

The closed form formula is computationally efficient and we can use it to find out which simulation method is better in a particular setting when the closed form solution is not available as in the case of American option. The calibration problem using the option price developed in this paper is an important issue for further research.

\section{Appendix 1}

Proof of Theorem 1. Since the arbitrage price of the European option is the discounted expected value of $X_{t}$ under the equivalent martingale measure $Q$, we have

$$
V_{i}(T, K, r)=E^{Q}\left[e^{-r T}\left(X_{T}-K\right)^{+} \mid \varepsilon(0)=i\right] .
$$

We will use $E$ to denote $E^{Q}$ for simplicity in the following argument. Recalling that under the risk neutral probability measure $Q$,

$$
X_{t}=X_{0} \exp \left(\int_{0}^{t}\left(r-\frac{1}{2} \sigma_{\varepsilon(s)}^{2}\right) d s+\int_{0}^{t} \sigma_{\varepsilon(s)} d W_{s}^{Q}\right) .
$$


We need to find the distribution of $X_{t}$. Let $Y_{t}=\ln X_{t}$, then

$$
Y_{t}=Y_{0}+\int_{0}^{t}\left(r-\frac{1}{2} \sigma_{\varepsilon(s)}^{2}\right) d s+\int_{0}^{t} \sigma_{\varepsilon(s)} d W_{s}^{Q} .
$$

Let $T_{i}$ be the total amount of time between 0 and $T$ such that $\varepsilon(t)=0$, starting from state $i$. Consider the probability density function $f_{i}(t, T)$ of $T_{i}$. Let $\mathcal{F}_{\varepsilon}(0)$ be the $\sigma$-field generated by $\varepsilon(0)$. Then

$$
\begin{aligned}
V_{i}(T, K, r) & =E\left[e^{-r T}\left(X_{T}-K\right)^{+} \mid \varepsilon(0)=i\right] \\
& =e^{-r T} E\left[E\left[\left(X_{T}-K\right)^{+} \mid T_{i}\right] \mid \varepsilon(0)=i\right] \\
& =e^{-r T} E_{i}\left[E\left[\left(X_{T}-K\right)^{+} \mid T_{i}\right] \mid \mathcal{F}_{\varepsilon}(0)=i\right] \\
& =e^{-r T} \int_{0}^{\infty} \int_{0}^{T} \frac{y}{y+K} \phi(\ln (y+K), m(t), v(t)) f_{i}(t, T) d t d y
\end{aligned}
$$

where

$$
\phi(x, m(t), v(t))=\frac{1}{\sqrt{2 \pi v(t)}} \exp \left[-\frac{(x-m(t))^{2}}{2 v(t)}\right]
$$

is the normal density function with mean $m(t)$ and variance $v(t)$. By (A.1), it is easy to see that

$$
\begin{aligned}
m(t) & =\ln (X(0))+\left(r T-\frac{1}{2} v(t)\right), \\
v(t) & =\left(\sigma_{0}^{2}-\sigma_{1}^{2}\right) t+\sigma_{1}^{2} T .
\end{aligned}
$$

Note that $f_{i}(t, T) d t=P\left(\int_{0}^{T} \chi_{0}(\varepsilon(s)) d s \in d t \mid \varepsilon(0)=i\right)$, where $\chi_{0}$ is the indicator function of state 0 . Let

$$
\begin{aligned}
\psi_{i}(r, T) & =E\left[e^{-r \int_{0}^{T} \chi_{0}(\varepsilon(s)) d s} \mid \varepsilon(0)=i\right] \\
& :=L_{r}\left(f_{i}(\cdot, T)\right) .
\end{aligned}
$$

By considering the two events $\left\{\tau_{i}>t\right\}$ and $\left\{\tau_{i} \leq t\right\}, i=0,1$, we have

$$
\begin{aligned}
& \psi_{0}(r, T)=e^{-r T} e^{-\lambda_{0} T}+\int_{0}^{T} e^{-\lambda_{0} u} \lambda_{0} \psi_{1}(r, T-u) e^{-r u} d u, \\
& \psi_{1}(r, T)=e^{-\lambda_{1} T}+\int_{0}^{T} e^{-\lambda_{1} u} \lambda_{1} \psi_{0}(r, T-u) d u .
\end{aligned}
$$

Taking Laplace transforms with respect to $T$, and writing

$$
\begin{aligned}
L_{s}\left(\psi_{i}(r, \cdot)\right) & =L_{s}\left[L_{r}\left(f_{i}(\cdot, T)\right)(r, \cdot)\right] \\
& :=\hat{\psi}_{i}(r, s),
\end{aligned}
$$


which can be solved for $\hat{\psi}_{i}(r, s)$ and yield

$$
\begin{aligned}
& \hat{\psi}_{0}(r, s)=\frac{s+\lambda_{0}+\lambda_{1}}{s^{2}+s \lambda_{1}+s \lambda_{0}+r s+r \lambda_{1}}, \\
& \hat{\psi}_{1}(r, s)=\frac{r+s+\lambda_{0}+\lambda_{1}}{s^{2}+s \lambda_{1}+s \lambda_{0}+r s+r \lambda_{1}} .
\end{aligned}
$$

Employing the inverse Laplace transform with respect to $r$, we have

$$
L_{r}^{-1}\left(\hat{\psi}_{0}(r, s)\right)(w, \cdot)=\frac{s+\lambda_{0}+\lambda_{1}}{s+\lambda_{1}} \exp \left(-\frac{s\left(s+\lambda_{0}+\lambda_{1}\right)}{s+\lambda_{1}} w\right) .
$$

Suppose the inverse Laplace transform of $f(s)$ is $F(t)$, then we have the following

$$
\begin{gathered}
L^{-1}[f(s+a)]=e^{-a t} F(t), \\
L^{-1}\left[e^{-a s} f(s)\right]=F(t-a) \chi(t-a),
\end{gathered}
$$

where $\chi$ is the step function

$$
\chi(t)= \begin{cases}0, & \text { if } t<0 \\ 1, & \text { if } t \geq 0\end{cases}
$$

Taking the inverse Laplace transform with respect to $s$ and applying (A.3) we have

$$
\begin{aligned}
& L_{s}^{-1}\left[L_{r}^{-1}\left(\hat{\psi}_{0}(r, s)\right)(w, \cdot)\right](\cdot, v) \\
& =L_{s}^{-1}\left[\frac{s+\lambda_{0}+\lambda_{1}}{s+\lambda_{1}} \exp \left(-\frac{s\left(s+\lambda_{0}+\lambda_{1}\right)}{s+\lambda_{1}} w\right)\right](\cdot, v) \\
& =e^{-\lambda_{1} v} L_{s}^{-1}\left[\frac{s+\lambda_{0}}{s} \exp \left(-\frac{\left(s-\lambda_{1}\right)\left(s+\lambda_{0}\right)}{s} w\right)\right](\cdot, v) \\
& =e^{-\lambda_{1} v} e^{\left(\lambda_{1}-\lambda_{0}\right) w} L_{s}^{-1}\left[\left(1+\frac{\lambda_{0}}{s}\right) \exp \left(-s w+\frac{\lambda_{0} \lambda_{1} w}{s}\right)\right](\cdot, v),
\end{aligned}
$$

Applying (A.4) to the right hand side of the last equation, we have

$$
\begin{aligned}
& L_{s}^{-1}\left[L_{r}^{-1}\left(\hat{\psi}_{0}(r, s)\right)(w, \cdot)\right](\cdot, v) \\
& =e^{-\lambda_{1} v} e^{\left(\lambda_{1}-\lambda_{0}\right) w} \chi(v-w) L_{s}^{-1}\left[e^{\frac{\lambda_{0} \lambda_{1} w}{s}}+\frac{\lambda_{0}}{s} e^{\frac{\lambda_{0} \lambda_{1} w}{s}}\right](\cdot, v-w) .
\end{aligned}
$$

Using the following facts concerning the Laplace transform of Bessel functions

$$
\begin{aligned}
L^{-1}\left(\frac{1}{s} e^{\frac{b}{s}}\right) & =I_{0}(2 \sqrt{b t}), \\
L^{-1}\left(e^{\frac{b}{s}}-1\right) & =\sqrt{\frac{b}{t}} I_{1}(2 \sqrt{b t}),
\end{aligned}
$$


on the preceding equation, we obtain

$$
\begin{aligned}
f_{0}(w, v)= & e^{-\lambda_{0} v} \delta_{0}(v-w)+e^{-\lambda_{1}(v-w)-\lambda_{0} w} \chi(v-w)\left[\lambda_{0} I_{0}\left(2\left(\lambda_{0} \lambda_{1} w(v-w)\right)^{1 / 2}\right)\right. \\
& \left.+\left(\frac{\lambda_{0} \lambda_{1} w}{v-w}\right)^{1 / 2} I_{1}\left(2\left(\lambda_{0} \lambda_{1} w(v-w)\right)^{1 / 2}\right)\right],
\end{aligned}
$$

where $\delta_{0}$ represents unit point mass at 0 . It is easy to verify that

$$
L^{-1}(1)=\delta_{0},
$$

that is, the inverse Laplace transform of 1 is unit point mass at 0 .

Similarly, take the inverse Laplace transformation of $\hat{\psi}_{1}$ with respect to $r$ to obtain

$$
L_{r}^{-1}\left(\hat{\psi}_{1}(r, s)\right)(w, \cdot)=\frac{\lambda_{1}\left(s+\lambda_{0}+\lambda_{1}\right)}{\left(s+\lambda_{1}\right)^{2}} \exp \left(-s w \frac{s+\lambda_{0}+\lambda_{1}}{s+\lambda_{1}}\right)+\frac{\delta_{0}(w)}{s+\lambda_{1}} .
$$

By (A.3), the inverse Laplace transform of preceding equation with respect to $s$ entails

$$
\begin{aligned}
& L_{s}^{-1}\left[L_{r}^{-1}\left(\hat{\psi}_{1}(r, s)\right)(w, \cdot)\right](\cdot, v) \\
& =e^{-\lambda_{1} v} L_{s}^{-1}\left[\frac{\lambda_{1}\left(s+\lambda_{0}\right)}{s^{2}} \exp \left(-\frac{\left(s-\lambda_{1}\right)\left(s+\lambda_{0}\right)}{s} w\right)+\frac{\delta_{0}(w)}{s}\right] .
\end{aligned}
$$

Rearranging the right hand side of the preceding equation yields

$$
\begin{aligned}
& e^{-\lambda_{1} v} \delta_{0}(w)+e^{-\lambda_{1} v} L_{s}^{-1}\left[\left(\frac{\lambda_{1}}{s}+\frac{\lambda_{0} \lambda_{1}}{s^{2}}\right) \exp \left(-s w+\left(\lambda_{1}-\lambda_{0}\right) w+\frac{\lambda_{0} \lambda_{1}}{s}\right)\right] \\
& =e^{-\lambda_{1} v} \delta_{0}(w)+e^{-\lambda_{1} v} e^{\left(\lambda_{1}-\lambda_{0}\right) w} L_{s}^{-1}\left[e^{-s w}\left(\frac{\lambda_{1}}{s}+\frac{\lambda_{0} \lambda_{1}}{s^{2}}\right) \exp \left(\frac{\lambda_{0} \lambda_{1}}{s}\right)\right] .
\end{aligned}
$$

By (A.4) and the fact that

$$
L^{-1}\left(\frac{1}{s^{2}} e^{\frac{b}{s}}\right)=\sqrt{\frac{t}{b}} I_{1}(2 \sqrt{b t})
$$

we arrive at

$$
\begin{aligned}
f_{1}(w, v)= & e^{-\lambda_{1} v} \delta_{0}(w)+e^{-\lambda_{1}(v-w)-\lambda_{0} w} \chi(v-w)\left[\lambda_{1} I_{0}\left(2\left(\lambda_{0} \lambda_{1} w(v-w)\right)^{1 / 2}\right)\right. \\
& \left.+\left(\frac{\lambda_{1} \lambda_{0}(v-w)}{w}\right)^{1 / 2} I_{1}\left(2\left(\lambda_{0} \lambda_{1} w(v-w)\right)^{1 / 2}\right)\right] .
\end{aligned}
$$

Substituting $(T, t)$ for $(v, w)$ in (A.5) and (A.6), the proof of Theorem 1 is completed.

\section{Appendix 2}




$$
\begin{aligned}
& f_{2}(w, u, v) \\
& =\delta_{0}(w) \delta_{0}(u) \exp \left(-\lambda_{2} v\right)+\lambda_{2} \delta_{0}(w) \exp \left(u \lambda_{2}-u \lambda_{1}\right) \exp \left(-\lambda_{2} v\right) \chi(v-u) \\
& \times I_{0}\left(2 \sqrt{u \lambda_{2}\left(\lambda_{1}-\lambda_{10}\right)} \sqrt{v-u}\right) \\
& +\lambda_{2}\left(\lambda_{1}-\lambda_{10}\right) \delta_{0}(w) \exp \left(u \lambda_{2}-u \lambda_{1}\right) \exp \left(-\lambda_{2} v\right) \chi(v-u) \sqrt{\frac{v-u}{u \lambda_{2}\left(\lambda_{1}-\lambda_{10}\right)}} \\
& \times I_{1}\left(2 \sqrt{u \lambda_{2}\left(\lambda_{1}-\lambda_{10}\right)} \sqrt{v-u}\right) \\
& +I_{0}\left(2 \sqrt{\lambda_{0} \lambda_{10} w} \sqrt{u}\right)\left(\lambda_{2} \lambda_{10} \exp \left(-w \lambda_{0}-u \lambda_{1}-(v-w-u) \lambda_{2}\right)\right) \chi(v-w-u) \\
& \times I_{0}\left(2 \sqrt{u \lambda_{2}\left(\lambda_{1}-\lambda_{10}\right)} \sqrt{v-w-u}\right) \\
& +\sqrt{\frac{u}{\lambda_{0} \lambda_{10} w}} I_{1}\left(2 \sqrt{\lambda_{0} \lambda_{10} w} \sqrt{u}\right)\left(\lambda_{0} \lambda_{10} \lambda_{2} \exp \left(-w \lambda_{0}-u \lambda_{1}-(v-w-u) \lambda_{2}\right)\right) \\
& \times \chi(v-w-u) I_{0}\left(2 \sqrt{u \lambda_{2}\left(\lambda_{1}-\lambda_{10}\right)} \sqrt{v-w-u}\right) \\
& +\lambda_{0} \lambda_{1}^{2} \lambda_{2} \frac{\lambda_{10}}{\lambda_{1}}\left(1-\frac{\lambda_{10}}{\lambda_{1}}\right) \exp \left(-w \lambda_{0}-u \lambda_{1}-(v-w-u) \lambda_{2}\right) \chi(v-w-u) \\
& \times \sqrt{\frac{v-w-u}{u \lambda_{2}\left(\lambda_{1}-\lambda_{10}\right)}} I_{1}\left(2 \sqrt{u \lambda_{2}\left(\lambda_{1}-\lambda_{10}\right)} \sqrt{v-w-u}\right) . \\
& f_{1}(w, u, v) \\
& =\delta_{0}(w) \exp \left(-u \lambda_{1}+u \lambda_{2}\right) \exp \left(-\lambda_{2} v\right) \chi(v-u) \\
& \times\left[\frac{\sqrt{\left(\lambda_{2}\left(\lambda_{1}-\lambda_{10}\right) u\right)} I_{1}\left(2 \sqrt{\lambda_{2}\left(\lambda_{1}-\lambda_{10}\right) u} \sqrt{v-u}\right)}{\sqrt{v-u}}+\delta_{0}(v-u)\right] \\
& +\delta_{0}(w) \exp \left(-u \lambda_{1}+u \lambda_{2}\right)\left(\lambda_{1}-\lambda_{10}\right) \exp \left(-\lambda_{2} v\right) \chi(v-u) \\
& \times I_{0}\left(2 \sqrt{\lambda_{2}\left(\lambda_{1}-\lambda_{10}\right) u} \sqrt{v-u}\right)+\lambda_{10} \exp \left(w \lambda_{2}+u \lambda_{2}-w \lambda_{0}-u \lambda_{1}\right) \\
& \times I_{0}\left(2 \sqrt{w \lambda_{10} \lambda_{0} u}\right) \exp \left(-\lambda_{2} v\right) \chi(v-w-u) \\
& \times\left(\frac{\sqrt{\lambda_{2}\left(\lambda_{1}-\lambda_{10}\right) u} I_{1}\left(2 \sqrt{\lambda_{2}\left(\lambda_{1}-\lambda_{10}\right) u} \sqrt{v-w-u}\right)}{\sqrt{v-w-u}}+\delta_{0}(v-w-u)\right) \\
& +\lambda_{1} \lambda_{10}\left(1-\frac{\lambda_{10}}{\lambda_{1}}\right) \lambda_{0} \sqrt{\frac{u}{w \lambda_{10} \lambda_{0}}} I_{1}\left(2 \sqrt{w \lambda_{10} \lambda_{0} u}\right) \exp \left(w \lambda_{2}+u \lambda_{2}-w \lambda_{0}-u \lambda_{1}\right) \\
& \times \exp \left(-\lambda_{2} v\right) \chi(v-w-u) I_{0}\left(2 \sqrt{\lambda_{2}\left(\lambda_{1}-\lambda_{10}\right) u} \sqrt{v-w-u}\right) \\
& +\lambda_{10} \lambda_{0} \sqrt{\frac{u}{w \lambda_{10} \lambda_{0}}} I_{1}\left(2 \sqrt{w \lambda_{10} \lambda_{0} u}\right) \exp \left(w \lambda_{2}+u \lambda_{2}-w \lambda_{0}-u \lambda_{1}\right) \exp \left(-\lambda_{2} v\right) \\
& \times \chi(v-w-u)\left(\frac{\sqrt{\lambda_{2}\left(\lambda_{1}-\lambda_{10}\right) u} I_{1}\left(2 \sqrt{\lambda_{2}\left(\lambda_{1}-\lambda_{10}\right) u} \sqrt{v-w-u}\right)}{\sqrt{v-w-u}}\right. \\
& \left.+\delta_{0}(v-w-u)\right) .
\end{aligned}
$$




\section{Acknowledgements}

The research was partially supported by a grant from National Center for Theoretical Sciences. The research of Cheng-Der Fuh was partially supported by NSC 92-2118-M-001-032. The research of Inchi Hu was partially supported by a grant from Hong Kong Research Grant Council.

\section{References}

Anderson, T. G. (1996). Return volatility and trading volume: an information flow interpretation of stochastic volatility. Journal of Finance 51, 169-204.

Avellaneda, M., Levy, A. and Parás, A. (1995). Pricing and hedging derivative securities in markets with uncertain volatilities. Applied Mathematical Finance 2, 73-88.

Bittlingmayer, G. (1998). Output, stock volatility and political uncertainty in a natural experiment: Germany, 1880-1940. Journal of Finance 53, 22432257.

Black, F. and Scholes, M. (1973). The pricing of options and corporate liabilities. Journal of Political Economy 81, 637-654.

Bollerslev, T. (1986). Generalized autoregressive conditional heteroskedasticity. Journal of Econometrics 31, 307-327.

Bollerslev, T. (1987). A conditionally heteroskedastic time series model for speculative prices and rates of return. Review of Economics and Statistics 69, 542-547.

Choudhury, G. L., Lucantoni, D. M. and Whitt, W. (1994). Multidimensional transform inversion with applications to the transient M/G/1 queue. Annals of Applied Probability 4, 719-740.

Cox, J. C., Ross, S. A. and Rubinstein, M. (1979). Option pricing: a simplified approach. Journal of Financial Economics 7, 229-263.

David, A. (1997). Fluctuating confidence in stock markets: implications for returns and volatility. Journal of Financial and Quantitative Analysis 32, 427-462.

Detemple, J. B. (1991). Further results on asset pricing with incomplete information. Journal of Economic Dynamics and Control 15, 425-453. 
Di Masi, G. B., Kabanov, Y. M. and Runggaldier, W. J. (1994). Mean-variance hedging of options on stocks with Markov volatility. Theory of Probability and Its Applications 39, 173-181.

Diebold, F. X. and Inoue, A. (2001). Long memory and regime switching. Journal of Econometrics 105, 131-159.

Duan J. C. (1995). The GARCH option pricing model. Mathematical Finance $\mathbf{5}, 13-32$.

Duan, J. C., Popova, I. and Ritchken, P. (2002). Option pricing under regime switching. Qunatitative Finance 2, 116-132.

Duffie, D. (1987). Stochastic equilibria with incomplete financial markets. Journal of Economic Theory 41, 405-416.

Duffie, D. and Huang, C. F. (1986). Multiperiod security markets with differential information. Journal of Mathematical Economics 15, 283-303.

Engle, R. F. (1982). Autoregressive conditional heteroscedasticity with estimates of the variance of United Kingdom inflation. Econometrics 50, 9871007.

Föllmer, H. and Sondermann, D. (1986). Hedging of nonredundant contingent claims. In Contributions to Mathematical Economics in Honor of Gérard Debreu (Edited by W. Hildenbrand and A. Mas-Colell), 205-223. NorthHolland, Amsterdam, New York.

Grorud, A. and Pontier, M. (1998). Insider trading in a continuous time market model. International Journal of Theoretical and Applied Finance 1, 331347.

Guilaume, D. M., Dacorogna, M., Davé, R., Muller, U., Olsen, R. and Pictet, P. (1997). From the bird's eye to the microscope, a survey of stylized facts of the intra-daily foreign exchange market. Finance and Stochastics 1, 95-129.

Guo, X. (2001). Information and option pricing. Quantitative Finance 1, 38-44.

Hamilton, J. D. (1988). Rational-expectations econometric analysis of changes in regime: an investigation of term structure of interest rates. Journal of Economic Dynamics and Control 12, 385-423.

Hamilton, J. D. (1989). A new approach to the economic analysis of nonstationary time series and the business cycle. Econometrica 57, 357-384. 
Hamilton, J. D. and Susmel, R. (1994). Autoregressive conditional heteroscedasticity and changes in regime. Journal of Econometrics 64, 307-333.

Heston, S. L. (1993). A closed-form solution for options with stochastic volatility with applications to bond and currency options. Review of Financial Studies 6, 327-343.

Hull, J. and White, A. (1987). The pricing of options on assets with stochastic volatility. Journal of Finance 2, 281-300.

Karatzas, I. and Pikovsky, I. (1996). Anticipative portfolio optimization. Advances in Applied Probability 28, 1095-1122.

Maghrebi, N., Kim, M. S. and Nishina, K. (2007). The KOSPI200 implied volatility index: evidence of regime switches in volatility expectations. Asia-Pacific Journal of Financial Studies 36, 163-187.

McKean, H. P. (1969). Stochastic Integrals. Academic Press, New York.

Ross, S. A. (1989). Information and volatility, the no-arbitrage martingale approach to timing and resolution irrelevancy. Journal of Finance 44, 1-8.

Schweizer, M. (1991). Option hedging for semimartingales. Stochastic Processes and their Applications 37, 339-363.

So, M. K. P., Lam, K. and Li, W. K. (1998). A stochastic volatility model with Markov switching. Journal of Business \& Economic Statistics 16, 244-253.

Stein, E. M. and Stein, C. J. (1991). Stock prices distribution with stochastic volatility, an analytic approach. Review of Financial Studies 4, 727-752.

Turner, C. M., Startz, R. and Nelson, C. R. (1989). A Markov model of heteroscedasticity, risk, and learning in the stock market. Journal of Financial Economics 25, 3-22.

Veronesi, P. (1999). Stock market overreaction to bad news in good times: a rational expectations equilibrium model. Review of Financial Studies 12, 975-1007.

Wiggins, J. B. (1987). Option values under stochastic volatility: Theory and empirical evidence. Journal of Financial Economics 19, 351-372. 
Fuh, Cheng-Der

Graduate Institute of Statistics

National Central University

Jhong-Li 320,Taiwan

stcheng@stat.sinica.edu.tw

Kwok Wah Remus Ho

Department of Statistics

Chinese University of Hong Kong

Shatin, Hong Kong

kwho@sta.cuhk.edu.hk

Inchi $\mathrm{Hu}$

Department of ISOM

Hong Kong University of Science and Technology

Clear Water Bay, Kowloon, Hong Kong

imichu@ust.hk

Ren-Her Wang

Department of Banking and Finance

Tamkang University

New Taipei 251, Taiwan

138230@mail.tku.edu.tw 